\title{
Transabdominal ultrasound screening for early ovarian cancer
}

\author{
Stuart Campbell, Vijay Bhan, Patrick Royston, Malcolm I Whitehead, William P Collins
}

\begin{abstract}
Objective-To assess the value of ultrasonography in a screening procedure for early ovarian cancer.

Design-Prospective study of at least 5000 self referred women without symptoms of ovarian cancer. Each woman was scheduled to undergo three annual screenings (consisting of one or more scans) to detect grossly abnormal ovaries or non-regressing masses.
\end{abstract}

Setting-The ovarian screening clinic at King's College Hospital, London.

Subjects-5479 Self referred women without symptoms (aged 18-78, mean age 52).

Interventions - Women with a positive result on screening were referred for laparoscopy or laparotomy, or both.

Main outcome measures-Findings at surgery and from histology of abnormal ovaries.

Results - A total of 14594 screenings (15977 scans) were performed. A positive result was obtained at 338 screens $(2 \cdot 3 \%)$ comprising 326 subjects (5.9\%). Five patients with primary ovarian cancer (four stage Ia, one stage Ib; two at first screening three at second) were identified (prevalence $0.09 \%$ ). An additional four patients had metastatic ovarian cancer (three at first screening, one at second). The apparent detection rate was $100 \%$. It was not possible to differentiate between the ultrasonic appearance of early malignant and benign tumours. The rate of false positive results for primary ovarian cancer was $3.5 \%$ at the first screening, $1.8 \%$ at the second, and $1 \cdot 2 \%$ at the third. Overall the rate of false positive results was $2.3 \%$; the specificity was $97.7 \%$ and the predictive value of a positive result on screening was $1 \cdot 5 \%$. The odds that a positive result on screening indicated the presence of an ovarian tumour, any ovarian cancer, or primary ovarian cancer were about one to two, one to 37 , and one to 67 respectively.

Conclusion-Ultrasonography can be used to screen women without symptoms for persistent ovarian masses that will include early ovarian cancer.

\section{Introduction}

Ovarian cancer is an insidious and intractable disease. The incidence in the United Kingdom increases with age to about 50 cases per 100000 women per year, and the cumulative risk up to 75 is $1 \cdot 3 \% .^{12}$ The disease is responsible for $4-5 \%$ of deaths in women below 60 . Currently less than $30 \%$ of primary cancers are confined to the ovaries at the time of diagnosis and the overall five year survival rate is less than $25 \%$. Consequently there is an urgent need for an effective, practical procedure to detect the disease at an early stage when women are without symptoms and the five year survival rate is greater than $80 \% .^{34}$

The measurement of a tumour marker (CA-125) in serum has been proposed as the initial stage of a screening procedure for early ovarian cancer, ${ }^{5}$ but more recent data suggest that the detection rate may be inadequate. ${ }^{6}$ For the past eight years we have investigated the use of real time pelvic ultrasonography as the first stage of a screening procedure for early ovarian neoplasms. ${ }^{7}$ Initially we showed that ovarian size and morphology assessed by ultrasound examination agreed well with results obtained by direct measurement and observation at laparotomy. Subsequently, the method of assessing ovarian morphology was pubished in more detail together with some preliminary screening results. ${ }^{8}$ Recently an encouraging report from another group has shown the potential value of ultrasonography for the detection of ovarian cancer in women attending an outpatient clinic. ${ }^{9}$ We describe the results of a large prospective study of an ultrasonography based screening procedure for early ovarian cancer in relation to menopausal state in self referred women over 44 without symptoms.

\section{Subjects and methods}

The plan was to screen at least 5000 self referred women without symptoms annually for three years. Women were made aware of the study by advertisements placed within King's College Hospital or in the local or national press, and any woman aged 45 or more could request a pelvic scan without the need for a medical referral. At each screening the women were classified as being either premenopausal (a menstrual period within the previous 12 months), artificially postmenopausal (due to hysterectomy with ovarian conservation or in one case to radiation treatment), or naturally postmenopausal (age greater than 35 and at least one elapsed year since the last menstrual period). Occasionally we recruited a woman who was under 44 (about $4 \%$ of the total) because of a personal or family history of cancer. One woman (aged 48) did not have a uterus at birth and had only one ovary, which was normal. Before examination the women completed a questionnaire designed to provide demographic data and a relevant personal and family medical history. An analysis of the distribution of variates for social class, marital state, parity, and history of infertility indicated that the self referred group (between the ages of 45 and 60 ) were similar to the general population of comparable age according to data from the Office of Population Censuses and Surveys over the period of the study.

Each screening consisted of one or more ultrasound examinations (scans). The women were instructed to drink a litre of fluid one hour before each scan. A full bladder displaces intestinal loops and provides a sonic window that enhances inspection of the pelvic organs. ${ }^{10}$ The ovaries were scanned transabdominally in the transverse and longitudinal planes with a Diasonics 100 mechanical sector scanner (Sonotron, Bedford) using a $3.5 \mathrm{MHz}$ transducer. Up to 30 women a day 
were scanned. A morphologically normal ovary gives an image with a smooth ovoid outline and a uniform, low level echogenicity similar to that of the myometrium. ${ }^{8}$

The maximum transverse $\left(\mathrm{D}_{1}\right)$, anteroposterior $\left(D_{2}\right)$, and longitudinal $\left(D_{3}\right)$ diameters of both ovaries were measured. Ovarian volumes were estimated according to the formula: volume $=(\pi / 6) \times D_{1} \times D_{2} \times$ $D_{3}$. At each screening the result of a scan was regarded as positive if any of three criteria was considered to be abnormal: ovarian morphology (hyperechogenicity or hypoechogenicity), outline (irregular), or volume (> about $20 \mathrm{ml}$ ). Women with a normal (negative) result on scanning were screened again about one year later. Those with an abnormal (positive) result were rescanned after three to eight weeks to exclude transient changes in ovarian morphology or size. If the result of the scan was still positive the woman was informed about the nature and possible significance of the findings. The information was sent to the appropriate general practitioner with a recommendation that the patient should be referred for laparoscopy or laparotomy, or both. Ostensibly abnormal ovarian tissues removed at surgery were sent for histological examination. The stage of the disease was estimated by the surgeon, and the final diagnosis was based on the report from the histologist. If the scan produced an equivocal result the woman was rescanned until the diagnosis was definitive or a decision had been made. All women who had at least one ovary received a maximum of three screens. The protocol was approved by the ethics committee at King's College Hospital.

Histopathology-Tissues from abnormal ovaries were examined at the referral hospitals and histology reports were sent to the ovarian screening clinic at King's College Hospital. The masses were classified according to criteria recommended by the World Health Organisation. ${ }^{11}$ The groups were common epithelial tumours, sex cord stromal tumours, germ cell tumours, and tumour-like conditions. The main subdivisions of common epithelial tumours were labelled as serous, mucinous, endometrioid, or clear cell. The tumours were also classified as benign, borderline, or malignant. The tumour-like conditions were subclassified as simple cysts, solitary follicle cysts, corpus luteum cysts, surface epithelium inclusion cysts, or parovarian cysts. We were unable (from the pathology reports) to differentiate between endometriosis (a tumour-like condition) and an endometrioid tumour. Accordingly, all such conditions were classified as endometrioid tumours for this analysis. The stage of each primary ovarian cancer was determined from the operation records according to the revised recommendations of the International Federation of Gynaecologists and Obstetricians. ${ }^{12}$ At stage Ia the tumour was limited to one ovary and the capsule was intact. There was no tumour on the external surface of the ovary and no ascites was present. At stage Ib the same criteria applied to both ovaries.

Database and statistical analysis-A database was established using FoxBase running on an IBM PC/XT compatible microcomputer with a hard disk. A tape

TABLE I-Age and menopausal state of study population at first screening. Values are numbers (percentages) of women

\begin{tabular}{lrrrrrr}
\hline & \multicolumn{8}{c}{ Age (years) } \\
\cline { 2 - 8 } Menopausal state & $\leqslant 44$ & $45-49$ & $50-54$ & $55-59$ & $\geqslant 60$ & All ages \\
\hline Premenopausal & 184 & 1457 & 740 & 78 & 5 & $2464(45 \cdot 1)$ \\
Naturally postmenopausal & 9 & 222 & 766 & 792 & 553 & $2342(42 \cdot 8)$ \\
Artificially postmenopausal & 31 & 218 & 217 & 130 & 65 & $661(12 \cdot 1)$ \\
\hline Total & $224(4 \cdot 1)$ & $1897(34 \cdot 7)$ & $1723(31 \cdot 5)$ & $1000(18 \cdot 3)$ & $623(11 \cdot 4)$ & $5467(100)^{\star}$
\end{tabular}

*In all, 5479 women were studied: age was not recorded in 11 women and one woman did not have uterus at birth.
TABLE II - Numbers of scans undertaken at each screening

\begin{tabular}{crrrr}
\hline & \multicolumn{3}{c}{ Screen No } & \\
\cline { 2 - 4 } Scan No & \multicolumn{1}{c}{1} & \multicolumn{1}{c}{2} & 3 & Total \\
\hline 1 & 5198 & 4576 & 3894 & 13668 \\
2 & 203 & 230 & 217 & 650 \\
3 & 54 & 68 & 63 & 185 \\
4 & 13 & 23 & 14 & 50 \\
5 & 5 & 5 & 9 & 19 \\
6 & 3 & 5 & 1 & 9 \\
$7-12$ & 3 & 7 & 3 & 13 \\
\hline Total & 5479 & 4914 & 4201 & 14594 \\
\hline
\end{tabular}

streamer was used for back up of data. A suite of programs was developed to facilitate data entry, checking, and retrieval. The detection rate (sensitivity) of the procedure was the proportion of women with primary ovarian cancer who had a positive result on screening. This criterion could only be ascertained within the limitation of the study design - that is, we were unable to ascertain independently whether every patient who had a negative result on screening was free of ovarian cancer. For some analyses the number of women with primary or metastatic ovarian cancer was used. The rate of false positive results for a particular screen was the proportion of women with a positive result on screening who were free of ovarian cancer. Again, this value could only be determined within the limitation of the study design - that is, we were unable to ascertain by another method the number of women in the study who did not have ovarian cancer.

\section{Results}

A total of 5479 women were recruited and underwent the first screening; $4914(90 \%)$ attended for the second screening and $4201(77 \%)$ for the third. Table I shows the age and menopausal state of each woman at the first screening (first scan). Their mean age was 52 (range 18-78). Initially about half of the women were aged between 50 and 59 and similar proportions were premenopausal $(45 \%)$ and naturally postmenopausal $(43 \%)$. Twelve per cent had undergone an artificial menopause. The five women above 60 classified as premenopausal all had menstrual cycles at the start of hormone replacement treatment. At the second screening roughly $36 \%(1644 / 4568)$ of the women were premenopausal and $51 \%$ (2330) had had a natural menopause. At the third screening the proportion of premenopausal women had decreased to $28 \%$ (1099/3889) and the proportion of naturally postmenopausal women had increased to $58 \%$ (2256). Most women who did not attend for the second and third screenings were naturally postmenopausal and over 60 .

Screening and scan variables-The median interval between the first and second screenings was 614 days (range 289-1134 days). The corresponding interval between the second and third screenings was 564 days (range 214-1019 days). Table II shows the number of scans performed at each screening. Sixty six women with at least one abnormal ovary only had one scan at a particular screen because the clinical ultrasonographer recommended immediate surgical investigation. The median time interval between the first and final scans at the first screening was 32 days (range 4-756 days). The corresponding value at the second screening was 65 days (range 14-1099 days). At the third screening the median time interval increased to 95 days (range 6-1097 days).

Outcomes of screening-The proportion of women who had a negative result on screening (neither ovary seemed to be abnormal), a positive and negative result (at least one ovary possibly abnormal at the first scan but subsequently regarded as normal after one or more repeat scans; positive, negative), and a positive and 
positive result (positive result on screening and recommended for surgical investigations; positive, positive) is shown for each screening in table III. The proportion of positive, positive results dropped from $3 \cdot 6 \%$ at the first screening to $1 \cdot 2 \%$ at the third and the ratio of positive, negative results for positive, positive results rose sevenfold from $0 \cdot 7$ to $5 \cdot 2$.

TABLE III-Results at each screening. Values are numbers (percentages) of women

\begin{tabular}{crcc}
\hline & \multicolumn{3}{c}{ Result } \\
\cline { 2 - 4 } Screen No & Negative & Positive, negative* & Positive, positive \\
\hline $1(\mathbf{n}=5479)$ & $5145(93 \cdot 9)$ & $139(2 \cdot 5)$ & $195(3 \cdot 6)$ \\
$2(\mathbf{n}=4914)$ & $4568(93 \cdot 0)$ & $254(5 \cdot 2)$ & $92(1 \cdot 9)$ \\
$3(\mathbf{n}=4201)$ & $3889(92 \cdot 6)$ & $261(6 \cdot 2)$ & $51(1 \cdot 2)$ \\
\hline Total & & 654 & 338
\end{tabular}

* Result initially positive but abnormality disappeared on rescanning. fOne or more rescans showed abnormality and patient referred for surgical investigation.

Table IV shows for each screening the numbers of women with different combinations of results and the numbers not attending. Most women had negative results at all screenings or negative results followed by non-attendance.

Ovarian masses at operation-The median time

TABLE IV - Numbers of subjects with different combinations of results and non-attendance at each screening

\begin{tabular}{lllr}
\hline \multicolumn{1}{c}{ Screen } & & \multicolumn{1}{c}{$\begin{array}{c}\text { No of } \\
\text { subjects } \\
(\mathbf{n}=5479)\end{array}$} \\
\hline Negative & \multicolumn{1}{c}{2} & \multicolumn{1}{c}{3} & Negative \\
Negative & Negative & Negative & 4061 \\
Negative & Non-attendance & Non-attendance & 639 \\
Positive & Non-attendance & Non-attendance & 453 \\
Positive & Negative & Negative & 112 \\
Negative & Positive & Non-attendance & 64 \\
Negative & Negative & Positive & 45 \\
Negative & Positive & Negative & 23 \\
Positive & Negative & Non-attendance & 12 \\
Negative & Positive & Positive & 5 \\
Positive & Positive & Non-attendance & 4 \\
Positive & Positive & Negative & 2 \\
Positive & Negative & Positive & 1 \\
\hline
\end{tabular}

TABLE V-Characteristics of patients with ovarian cancer

\begin{tabular}{|c|c|c|c|c|c|}
\hline Case No & $\begin{array}{c}\text { Screen } \\
\text { No }\end{array}$ & $\begin{array}{c}\text { Age } \\
\text { (years) }\end{array}$ & Menopausal state & Histological diagnosis & Stage \\
\hline \multicolumn{6}{|c|}{ Primary cancer } \\
\hline 1 & 2 & 59 & Naturally postmenopausal & Mucinous cystadenocarcinoma borderline & Ia \\
\hline 2 & 1 & 53 & Naturally postmenopausal & Serous cystadenocarcinoma borderline & $\mathrm{Ib}$ \\
\hline 3 & $2^{\star}$ & 46 & Premenopausal & Endometrioid borderline & Ia \\
\hline 4 & 2 & 61 & Naturally postmenopausal & Serous papillary cystadenocarcinoma & Ia \\
\hline 5 & 1 & 60 & Naturally postmenopausal & Clear cell carcinoma & Ia \\
\hline \multicolumn{6}{|c|}{ Secondary cancer } \\
\hline 6 & 1 & 54 & Premenopausal & Breast & \\
\hline 7 & 2 & 58 & Naturally postmenopausal & Breast & \\
\hline 8 & 1 & 60 & Artificially postmenopausal & Colon & \\
\hline 9 & 1 & 49 & Artificially postmenopausal & Breast & \\
\hline
\end{tabular}

*Contralateral ovary removed after benign endometrioid tumour detected on first screening.

TABLE VI-Relation between histological classification of 267 ovarian masses removed at laparotomy and ovarian morphology observed by ultrasonography

\begin{tabular}{|c|c|c|c|c|c|c|}
\hline \multirow[b]{3}{*}{ Ovarian morphology } & \multicolumn{5}{|c|}{ Histological classification of tumours } & \multirow[b]{3}{*}{ Total } \\
\hline & \multirow{2}{*}{$\begin{array}{l}\text { "Tumour } \\
\text { like"» }\end{array}$} & \multirow{2}{*}{$\begin{array}{l}\text { Sex cord } \\
\text { stromal }\end{array}$} & \multirow{2}{*}{$\begin{array}{l}\text { Germ } \\
\text { cell }\end{array}$} & \multicolumn{2}{|c|}{ Epithelial } & \\
\hline & & & & Benign & Malignant $\dagger$ & \\
\hline Normal & 17 & 1 & 1 & 12 & & 31 \\
\hline Unilocular cyst & 79 & 4 & 3 & 49 & 5 & 140 \\
\hline Unilocular cyst or solid & 12 & 1 & 11 & 18 & 4 & 46 \\
\hline Multilocular cyst & 19 & 1 & & 12 & 3 & 35 \\
\hline Multilocular cyst or solid & 6 & & 1 & 7 & & 14 \\
\hline Solid & & 1 & & & & 1 \\
\hline Total & 133 & 8 & 16 & 98 & 12 & 267 \\
\hline
\end{tabular}

^See World Health Organisation classification."

tPrimary and secondary interval between the final scan at any screening and operation was 48 days (range 12-191 days). A total of 379 ovarian masses were found at operation in the 326 women (from 338 screens) who had a positive result on screening. There were six primary ovarian cancers (five patients, one having bilateral tumours) and six metastatic ovarian cancers (four patients, two having bilateral tumours). Thus nine women had ovarian cancer (the prevalence of all cancers was $0 \cdot 16 \%$; and the prevalence of primary cancer was $0 \cdot 09 \%$ ). Table $\mathrm{V}$ shows some details of the cases. All of the primary cancers were stage Ia or Ib. Most of the other classified ovarian masses were either tumour-like conditions $(50 \%)$ or benign epithelial tumours $(37 \%)$. A further $9 \%$ were either sex cord stromal or germ cell tumours. About $11 \%$ of all ovarian masses were unclassified but judged to be non-malignant by the surgeon, and the patient refused further investigation. A further $18 \%$ of the apparent masses ( 62 women) observed by ultrasonography had either resolved spontaneously or were not of ovarian origin at the time of operation. Twenty two of these women were premenopausal and 28 postmenopausal. Only 12 women $(3 \cdot 7 \%$ of those with a positive result on screening) were free of any pelvic disease at the time of surgical investigation.

Table VI shows the relation between various characteristics of ovarian morphology as observed by ultrasonography with the histological classification of tissues removed at operation. It was not possible to identify characteristics that were unique to the five early malignant tumours. An irregular outline was observed in $76 \%(101 / 133)$ of tumour-like conditions, $75 \%(6 / 8)$ of sex cord stromal tumours, $69 \%(11 / 16)$ of germ cell tumours, $70 \%$ (69/98) of benign epithelial tumours, and $67 \%(8 / 12)$ of malignant tumours (primary and secondary).

Evaluation of screening procedure-The screening procedure had a detection rate of $100 \%$ (within the limitation of the study design) with an overall rate of false positive results of $2 \cdot 3 \%$, as shown in table VII.

TABLE VII -False positive results of screening procedure for early primary ovarian cancer expressed per screen ${ }^{\star}$ and overall according to menopausal state

\begin{tabular}{lcccc}
\hline & \multicolumn{3}{c}{ False positive rate (\%) per screen } & \\
\cline { 2 - 4 } Menopausal state & 1 & 2 & 3 & Total \\
\hline Premenopausal & 3.5 & 1.6 & 1.3 & 2.4 \\
Naturally postmenopausal & 3.1 & 1.8 & 1.0 & 1.9 \\
Total $\dagger$ & 3.5 & 1.8 & 1.2 & 2.3 \\
\hline
\end{tabular}

Twelve subjects had positive results at two screenings (see table IV) Includes subjects who had artificial menopause.

There was a reduction in the rate of false positive results with each successive screening and the values were lower for women who were naturally postmenopausal. Table VIII summarises some of the criteria for evaluating the overall screening procedure - that is, all subjects, three screens combined. The odds against a positive screen result being associated with any ovarian tumour (by histological classification) were one to two-that is, one tumour per three cases. The odds fell

TABLE VIII-Some criteria for evaluating screening procedure (all subjects, three screens combined)

\begin{tabular}{lccccc}
\hline \multicolumn{1}{c}{$\begin{array}{c}\text { Ovarian } \\
\text { pathology }\end{array}$} & $\begin{array}{c}\text { Detection } \\
\text { rate } \\
(\%)\end{array}$ & $\begin{array}{c}\text { False } \\
\text { positives } \\
(\%)\end{array}$ & $\begin{array}{c}\text { Specificity } \\
(\%)\end{array}$ & $\begin{array}{c}\text { Positive } \\
\text { predictive } \\
\text { value } \\
(\%)\end{array}$ & Odds \\
\hline All masses & & & & $80 \cdot 8$ & $4 \cdot 2$ to 1 \\
All tumours & & & & 36.3 & 1 to 1.8 \\
All cancers & 100 & 2.3 & $97 \cdot 7$ & $2 \cdot 7$ & 1 to 37 \\
Primary cancer & 100 & 2.3 & $97 \cdot 7$ & 1.5 & 1 to 67 \\
\hline
\end{tabular}

*Against positive result on screening indicating presence of each type of pathology; odds = positive predictive value/(1 - positive predictive value $)$. 
to one to 37 for any ovarian cancer, and the values for primary ovarian cancer were one to 67 .

\section{Discussion}

To our knowledge this is the first report of a prospective study designed to assess the potential value of pelvic ultrasonography as a technique for the detection of early ovarian cancer in self referred women without symptoms who are representative of the general population between the ages of 45 and 60 . The low prevalence of the disease necessitated the study of at least 5000 women, and changes in ovarian morphology and volume were monitored by repeat scans. In addition, all subjects were scheduled to undergo three screenings to obtain more information about the prevalence of the disease in the study population and the most useful interval between screenings. Eventually 5479 women were monitored over eight years; 14594 screenings (15 977 scans) were undertaken and 29140 ovaries were examined. Some of the potential screening indices of ovarian cancer had not been described or were ill defined at the start of the study and had to be analysed retrospectively. These variates and new screening strategies will be the subjects of an additional publication. Accordingly, the persistence of abnormal ovarian morphology was the main criterion for the decision to refer women for further investigation. The results of the screening procedure are expressed either per screening or per patient because 92 women had an initially positive result at more than one screening. Of these, 12 women had a finally positive result at the first and second screenings.

In practice many of the women had to travel long distances to attend the scanning clinic and some underwent unilateral or bilateral oophorectomy as a consequence of the result on screening. For these and other reasons we were reassured that $90 \%$ of women who were screened initially attended for the second screening and $77 \%$ for the third - that is, compliance was good. The progressive increase in the ratio of initially positive and finally negative results to initially positive and finally positive results at the second and third screening probably reflects a change in the type of ovarian pathology because of previous interventions. It may also be due in part to the increased experience of the ultrasonographers or the changing menopausal state of the study population. Five subjects with primary ovarian cancer (all stage Ia or Ib) were detected (a notable result for a screening procedure), giving an overall prevalence for the disease in the study population of $0.09 \%$, which is about the proportion expected. Only one of the primary cancers could be detected by manual examination after ultrasonography. Three of the primary cancers were classified as borderline. This finding, together with the prevalence of the disease, may indicate the malignant potential of some borderline (or preinvasive) tumours. All of the women with primary ovarian cancer and three of the four women with metastatic ovarian cancer were alive at least two years after surgery. We currently have follow up data on $86 \%$ (4705) of the other women showing that they were free of any signs of ovarian cancer one year after the last screening or treatment. For these reasons we believe that the overall detection rate of the screening procedure and the predictive value of a negative test result were both probably $100 \%$ for this particular study, although the figure must of course decrease with time for both variables and will be the subject of further publications. It is thus particularly interesting that three primary cancers were detected at the second screening about 16,18 , and 22 months after the first. This finding suggests that screening every 12 to 18 months is necessary. Only a few benign tumours and no primary cancers were detected at the third screening. The importance of this finding may become apparent from detailed follow up studies and may have important implications for a screening programme.

We have already published data on the size of ovaries in postmenopausal women ${ }^{13}$ and ultrasound pictures of the primary cancers ${ }^{14}$ and related all ovarian masses that were found at surgery to the number of the screening and the menopausal state and age of the subjects. ${ }^{15}$ As indicated in table VI, where the results are expressed per ovary, we were unable to identify any morphological characteristics or patterns that could be used to differentiate between ovarian tumour-like conditions and tumours or between benign and early malignant tumours. The latter finding is disappointing in view of the more encouraging results reported by previous workers, ${ }^{1617}$ who mainly studied later stages of the disease in women with symptoms and excluded borderline tumours from their analyses. The finding of various masses at surgery in ultrasonically normal ovaries at the time of the last scan may be related to the time interval between the two observations or to the occasional use of an overtly enlarged ovary as a criterion for a positive result of screening.

The screening procedure gave a rate of false positive results for the detection of primary ovarian cancer of $3.5 \%$ at the first screening and $1 \cdot 2 \%$ at the third $(2 \cdot 3 \%$ overall). The reduction in the rate over successive screenings is probably due to the treatment of women with one or more persistent ovarian masses. The overall specificity of the screening procedure was $97.7 \%$, and the predictive value for a positive test result was $1.5 \%$ - that is, odds of one to 67 that a positive result was indicative of primary ovarian cancer. We believe, however, that these values must be interpreted in the knowledge that the odds for a positive result on screening indicating the presence of any ovarian tumour were one to two (because some of these tumours will constitute a health hazard); and for all ovarian cancers the odds were one to 37 . The rate of false positive results for primary ovarian cancer was lower for women who were naturally menopausal and the results from our more recent study suggest that the values for premenopausal women may be reduced by a careful consideration of the day of the menstrual cycle and the thickness of the endometrium in the presence of an ovarian mass. The rate of false positive results for any ovarian abnormality was only $0.6 \%$ at the first screening and $0.4 \%$ overall, and these figures will undoubtedly be lower in future studies owing to the experience gained and the technical developments in transvaginal ultrasonography (see accompanying paper $^{18}$ ). We believe that women will be attracted to a screening programme if the chances of detecting the disease at an early stage are high. Our results show that ultrasonography fulfils this criterion and future efforts will be directed toward reducing the rate of false positive results. There is still, however, the suggestion that apparently benign epithelial tumours may have an increased potential for malignancy compared with healthy tissues. If this possibility were to be substantiated the removal of these tumours would mean that ultrasound screening would aid the prevention as well as the detection of primary ovarian cancer.

We thank the Cancer Research Campaign for financial support, Dr R Goswamy for undertaking the initial ultrasound scans, Sisters R Battersby, V Hall, J Foxton, and A Davies for their nursing skills, Messrs L Schiphorst and C Harris for establishing the database, and Mrs J Monk for secretarial help. MIW was supported by the Imperial Cancer Research Fund. 1 Cramer DW. Epidemiologic and statistical aspects of gynecologic oncology
In: Knapp RC, Berkowitz RS, eds. Gynecologic oncology. New York: Macmillan, 1986:201-22. 
2 Beral V. The epidemiology of ovarian cancer. In: Sharp F, Soutter WP, eds. Ovarian cancer-the way ahead. Chichester: Wiley, 1987:21-31.

L Ono DL, Young RC. The natural history and treatment of ovain cancer. Ann Rev Med 1981:32:475-90.

+ Barker HRK. Ovarian cancer, diagnosis and management. Am 7 Obstet Gynecol 1984:150:910-6.

5 Jacobs I, Stabile I, Bridges J, et al. Multimodal approach to screening for ovarian cancer. Lancet 1988;i:268-71.

6 Zurawski VR, Orjaseter H, Andersen A, Jellum E. Elevated serum CA-125 levels prior to diagnosis of ovarian neoplasia: relevance for early detection of ovarian cancer. Int $f$ Cancer 1988;42:677-80

7 Campbell S, Goessens L, Goswamy R, Whitehead MI. Real-time ultrasonography for the determination of ovarian morphology and volume. A possible early screening test for ovarian cancer. Lancet 1982;i:425-6.

8 Goswamy RK, Campbell S, Whitehead MI. Screening for ovarian cancer. In: Campbell S, ed. Ultrasound in obstetrics and gynaecology: recent advances. London: Saunders, 1983:621-43. (Clinics in Obstetrics and Gynaecology 1983:10:621-43.

9 Andolf $\mathrm{E}$, Svalenius E, Astedt B. Ultrasonography for early detection of ovarian carcinoma. Brf Obstet Gynaecol 1986;93:1286-9.

10 Donald I. Use of ultrasonics in the diagnoses of abdominal swellings. Br Med $\mathcal{f}$ 1963;ii:1154-5.
11 Serov SF, Scully RE, Sobin LH. International histological classification of tumours. No 9 Histological typing of ovarian tumours. Geneva: World Health Organisation, 1973.

12 Creasman WT. Changes in FIGO staging. Obstet Gynecol 1987;70:138.

13 Goswamy RK, Campbell S, Royston JP, et al. Ovarian size in postmenopausal women. Br f Obstet Gynaecol 1988;95:795-801.

14 Bhan V, Campbell S. Ultraschall als Screening-Vertahren zur Entdeckung von Ovarialtumoren. Gynakologe 1986;19:135-41.

15 Bhan V, Amso N, Whitehead MI, Campbell S, Royston P, Collins WP. Characteristics of persistent ovarian masses in asymptomatic women. $\mathrm{Br}$. Obstet Gynaecol (in press).

16 Meire HB, Farrant P, Guha T. Distinction of benign from malignant ovarian cysts by ultrasound. Br f Obstet Gynaecol 1978;85:893-9.

17 Hermann UJ, Locker GW, Goldhirsh A. Sonographic patterns of ovarian tumors: prediction of malignancy. Obstet Gynecol 1987;69:777-81.

18 Bourne T, Campbell S, Steer C, Whitehead MI, Collins WP. Transvaginal colour flow imaging: a possible new screening technique for ovarian cancer. BrMed f 1989;299:1367-70.

(Accepted 10 October 1989)

\section{Transvaginal colour flow imaging: a possible new screening technique for ovarian cancer}

Department of Obstetrics and Gynaecology, King's College School of Medicine and Dentistry, London SE5 8RX

Thomas Bourne, MB, research registrar

Stuart Campbell, FRCOG, professor

Malcolm I Whitehead, FRCOG, senior lecturer William P Collins, DSC, professor

Hallam Medical Centre, London W1N 5LR Christopher Steer, MRCOG, research registrar

Correspondence to: Professor Collins.

\section{Thomas Bourne, Stuart Campbell, Christopher Steer, Malcolm I Whitehead, William P Collins}

\section{Abstract}

Objective-To assess whether changes in the intraovarian vasculature or blood flow impedance can be used to identify potentially malignant masses.

Design-Open, non-comparative prospective study.

Setting-Ovarian screening clinics at King's College Hospital and the Hallam Medical Centre.

Subjects -50 Women selected on the basis of their medical history and the result of a previous transvaginal ultrasound scan. Thirty women (10 premenopausal (scan taken on days 1 to 8 of the menstrual cycle) and 20 postmenopausal) had normal ovaries, and 20 had at least one ovary with an abnormal morphology or volume, or both.

Interventions - Women with a positive result on screening were referred for laparotomy.

Main outcome measures-Presence or absence of coloured areas (neovascularisation) and the pulsatility index within each ovary. The pulsatility index is a measure of the impedance to blood flow, a low value indicating decreased impedance and a high value increased impedance to blood flow.

Results-Two women with a positive result on screening had hydrosalpinges, 10 a benign tumour or a tumour-like condition, and eight primary ovarian cancers. No areas of neovascularisation were seen in the $\mathbf{3 0}$ women with morphologically normal ovaries and the two patients with hydrosalpinges; the pulsatility index ranged from $3 \cdot 1$ to $9 \cdot 4$. Similarly, nine patients (10 affected ovaries) with a nonmalignant mass had no signs of neovascularisation and the pulsatility index varied from $3 \cdot 2$ to $7 \cdot 0$. One patient with bilateral dermoid cysts containing nests of thyroid-like cells had vascular changes and pulsatility index values of 0.4 and $0 \cdot 8$. Seven patients (eight ovaries) with primary ovarian cancer (one stage IV, four stage III, and two stage Ia) showed clear evidence of neovascularisation and pulsatility index values were from 0.3 to $1 \cdot 0$. One patient with an intraepithelial serous cystadenocarcinoma in a small ovary ( $<5 \mathrm{ml}$ volume) had no signs of any vascular change and the pulsatility index was $5 \cdot 5$.

Conclusion-Transvaginal colour flow imaging may be used to identify potentially malignant ovarian masses and help elucidate the early stages of tumorigenesis. The routine application of this technique may reduce the rate of false positive results of an ultrasonography based screening procedure.

\section{Introduction}

Primary ovarian cancer affects about 5000 women in the United Kingdom each year. The five year survival rate depends on the stage of the disease at the time of diagnosis and ranges from less than $5 \%$ at stage IV to more than $80 \%$ at stage Ia or Ib. The overall mortality is more than $80 \%$ and a late diagnosis by traditional techniques is probably the main reason for the poor prognosis. ${ }^{12}$ The outcome may be improved by the introduction of new treatment regimens, but the development of procedures for the early detection of the disease is probably the best approach to achieve a reduction in the mortality.

We are investigating the use of pelvic ultrasonography as a screening procedure for early ovarian neoplasms. Initially Campbell et $\mathrm{al}$ showed that ovarian size and morphology as assessed by transabdominal ultrasound examination agreed well with results obtained by direct measurement and observation at laparotomy. ${ }^{3}$ Recently the results of a prospective study of 5479 self referred women without symptoms have been described in terms of the ovarian masses detected, ${ }^{+}$the value of the screening procedure over time (see previous paper ${ }^{s}$ ), and the development of new screening strategies entailing the use of defined changes in ovarian volume (S Campbell et al, unpublished data). Five primary ovarian cancers were detected at stage Ia or Ib, and evidence from a follow up study at least one year after the last screening showed that the detection rate was $100 \%$ within the limitation of the study design. A screening procedure based on the presence of abnormal ovarian morphology at the first scan and a defined volume change on rescanning would have given a false positive rate of $1.6 \%$ and a positive predictive value of $2.0 \%$-that is, the odds against a positive screen result indicating the presence of primary ovarian cancer were 1:50. This odds ratio is mainly due to the difficulty of distinguishing malignant tumours from benign masses, tumour-like conditions, or hydrosalpinges. We report the use of transvaginal ultrasonography with colour flow imaging for the detection of intraovarian 